\title{
Design and Analysis of Morpheus Lander Flight Control System
}

\author{
Jiann-Woei Jang ${ }^{1}$, Lee Yang ${ }^{2}$ and Mathew Fritz ${ }^{3}$ \\ The Charles Stark Draper Laboratory, Inc., Houston, TX, 77058 \\ and \\ Louis H. Nguyen ${ }^{4}$, Wyatt R. Johnson ${ }^{5}$ and Jeremy J. Hart ${ }^{6}$ \\ NASA/Johnson Space Center, Houston, TX, 77058
}

\begin{abstract}
The Morpheus Lander is a vertical takeoff and landing test bed vehicle developed to demonstrate the system performance of the Guidance, Navigation and Control (GN\&C) system capability for the integrated autonomous landing and hazard avoidance system hardware and software. The Morpheus flight control system design must be robust to various mission profiles. This paper presents a design methodology for employing numerical optimization to develop the Morpheus flight control system. The design objectives include attitude tracking accuracy and robust stability with respect to rigid body dynamics and propellant slosh. Under the assumption that the Morpheus time-varying dynamics and control system can be frozen over a short period of time, the flight controllers are designed to stabilize all selected frozen-time control systems in the presence of parametric uncertainty. Both control gains in the inner attitude control loop and guidance gains in the outer position control loop are designed to maximize the vehicle performance while ensuring robustness. The flight control system designs provided herein have been demonstrated to provide stable control systems in both Draper Ares Stability Analysis Tool (ASAT) and the NASA/JSC Trick-based Morpheus time domain simulation.
\end{abstract}

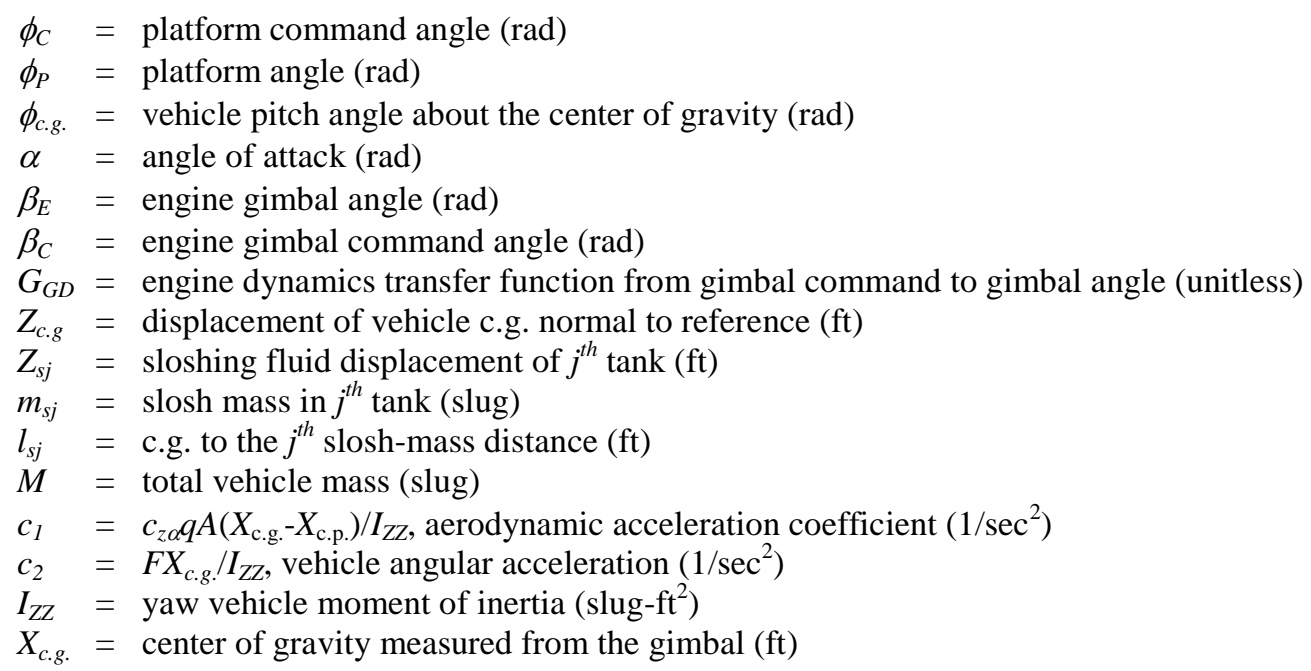

${ }^{1}$ Principal Member of the Technical Staff; Draper Laboratory, Houston TX, 77058: jang@ draper.com, AIAA Senior Member ${ }^{2}$ Senior Member of the Technical Staff; Draper Laboratory, Cambridge, MA, 02139: lee.yang@draper.com

${ }^{3}$ Member of the Technical Staff; Draper Laboratory, Houston, TX, 77058: mfritz@ draper.com

${ }^{4}$ NASA/Johnson Space Center, Houston, TX 77058: louis.h.nguyen@nasa.gov

${ }^{5}$ NASA/Johnson Space Center, Houston, TX 77058: wyatt.johnson-1@nasa.gov

${ }^{6}$ NASA/Johnson Space Center, Houston, TX 77058: jeremy.j.hart@ nasa.gov 
$X_{c . p .}=$ center of pressure measured from the gimbal (ft)

$S_{E}=$ engine $1^{\text {st }}$ moment about gimbal (slug- $\mathrm{ft}$ )

$I_{E} \quad=$ engine $2^{\text {nd }}$ moment about gimbal (slug- $\mathrm{ft}^{2}$ )

$k_{3}=(F-D) / M$, vehicle acceleration $\left(\mathrm{ft} / \mathrm{sec}^{2}\right)$

$k_{4}=F / M$, acceleration due to gimballing $\left(\mathrm{ft} / \mathrm{sec}^{2}\right)$

$k_{7}=C_{Z \alpha} q A / M$, acceleration due to aerodynamic forces $\left(\mathrm{ft} / \mathrm{sec}^{2}\right)$

$C_{Z \alpha}=$ rigid-body aerodynamic side force coefficient slope (unitless)

$q=$ dynamic pressure $\left(\mathrm{lbf} / \mathrm{ft}^{2}\right)$

$A=$ reference area $\left(\mathrm{ft}^{2}\right)$

$F \quad=$ gimbaled engine thrust (lbf)

$V_{w}=$ cross-wind velocity $(\mathrm{ft} / \mathrm{sec})$

$V=$ vehicle velocity $(\mathrm{ft} / \mathrm{sec})$

$z_{\beta i}=$ damping factor of the $i^{\text {th }}$ bending mode (unitless)

$\omega_{b i}=$ natural frequency of the $i^{\text {th }}$ bending mode $(\mathrm{rad} / \mathrm{sec})$

$M_{i}=$ generalized mass of the $i^{\text {th }}$ bending mode (unitless)

$\eta_{i}=$ bending displacement of the $i^{\text {th }}$ bending mode $\left(\operatorname{slug}^{1 / 2} \mathrm{ft}\right)$

$Y_{\beta i}=$ bending mode shape at gimbal $\left(\operatorname{slug}^{-1 / 2}\right)$

$Y_{\beta I}{ }^{\prime}=$ bending mode slope at gimbal $\left(\mathrm{rad}_{-\mathrm{slug}^{-1 / 2}} / \mathrm{ft}\right)$

$Y_{\phi i}{ }^{\prime}=$ bending mode slope at platform location $\left(\mathrm{rad}^{\prime} \mathrm{slug}^{-1 / 2} / \mathrm{ft}\right)$

$Y_{\dot{\phi} i}{ }^{\prime}=$ bending mode slope at rate gyro location $\left(\operatorname{rad}^{\prime} \operatorname{slug}^{-1 / 2} / \mathrm{ft}\right)$

$Y_{s i j}=$ deflection of the $i^{\text {th }}$ bending mode at the $j^{\text {th }}$ slosh mass location ( $\operatorname{slug}^{-1 / 2}$ )

$C_{Z \eta i}=$ wind bending force coefficient for the $i^{\text {th }}$ bending mode (lbf- slug ${ }^{-1 / 2} / \mathrm{rad}$ )

$n_{b}=$ number of bending modes

$n_{s}=$ number of slosh modes (determined by number of propellant tanks)

$\phi_{r} \quad=$ vehicle roll angle about the vehicle $\mathrm{x}$-axis $(\mathrm{rad})$

$\dot{\phi}_{r}=$ vehicle roll rate about the vehicle $\mathrm{x}$-axis $(\mathrm{rad} / \mathrm{s})$

\section{Introduction}

The Morpheus Lander is a vertical takeoff and landing test bed vehicle ${ }^{1}$. The prototype vehicle was manufactured and assembled at NASA's Johnson Space Center (JSC). The Morpheus Lander is used to demonstrate the system performance of the Guidance, Navigation and Control (GN\&C) system capability for the integrated autonomous landing and hazard avoidance system hardware and software. The Morpheus Lander consists of a throttleable main engine using liquid oxygen and liquid methane as propellant, four spherical aluminum tanks ( 2 for liquid methane and 2 for liquid oxygen) and the necessary avionics to perform powered descent and landing to provide realistic flight trajectories to demonstrate hazard detection and avoidance technology. The Morpheus flight control system utilizes Thrust Vector Control (TVC) of the main engine to control the vehicle Body pitch and yaw axes and fixed reaction control system thrusters to control the vehicle Body roll axis. The onboard GN\&C sensor suite includes a Global Positioning System (GPS) receiver, International Space Station (ISS) version of Honeywell's Space Integrated GPS/INS (SIGI), Litton LN-200 Inertial Measurement Unit (IMU) and an Acuity laser altimeter ${ }^{1}$. The Morpheus' primary payload is the Autonomous Landing Hazard Avoidance Technology (ALHAT), which will scan and detect hazards at the targeted landing site and interface with Morpheus' GN\&C system to autonomously avoid landing on any hazards (e.g. boulder or crater).

The Morpheus Lander has several characteristics, which must be taken into account in design. First, it is aerodynamically unstable because the vehicle's center of pressure is above its center of gravity. Second, the vehicle's mass, moment of inertia, aerodynamics and slosh characteristics vary greatly throughout the flight timeline. Third, the Morpheus flight control system must be robust to various mission profiles as shown in Figure 1. The outer guidance loop interacts with the inner attitude control loop and may result in instability if excessive guidance gains are used.

To achieve the desired performance and guaranteed robust stability for the Morpheus control system, the flight controller design can be divided into four phases: 
(1) Optimal rigid controller designs, which include phase plane controllers for roll channel and ProportionalIntegral-Derivative (PID) controllers for pitch and yaw channels.

(2) Robust guidance gain design to guarantee robust stability and adequate performance.

(3) Flex filter designs to guarantee robust stability and adequate performance.

(4) High fidelity nonlinear simulation to verify stability and performance for entire Morpheus control system.

These design phases are not necessarily performed in sequence. For example, guidance and controller designs can be combined into an iterative design process.

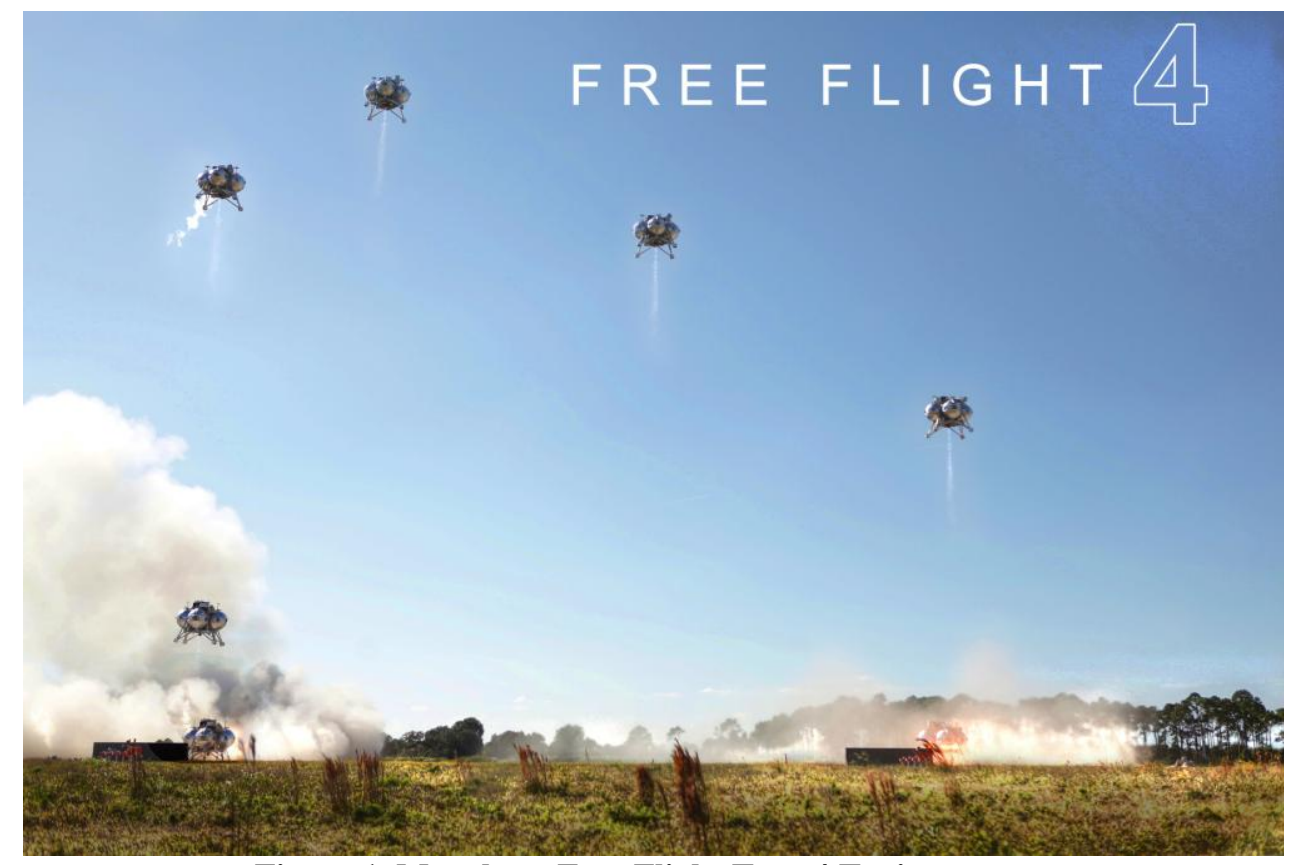

Figure 1. Morpheus Free Flight Test 4 Trajectory

A series of optimal guidance commands for each mission are designed for takeoff and ascent to altitude, hover and/or flight descent trajectory, and landing the Morpheus vehicle. The flight control system is designed to ensure stable response to guidance commands while minimizing trajectory deviations. The TVC system gimbals the main engine cone to minimize both pitch and yaw attitude errors. Slosh frequencies change over time during the entire flight. Flex filters in the flight control system are designed to minimize the slosh components in the error signals before they are sent to the attitude controller.

Digital filter designs and application have been extensively investigated in the literature ${ }^{2-4}$. The most popular analog filter design approaches include Butterworth, Chebyshev and elliptic filters. Direct digital filter design methodologies are also available in the literature ${ }^{3}$. These methodologies have been used for open-loop system designs by shaping filters to meet the open-loop performance specifications in the frequency domain. Unfortunately, most of the closed-loop system stability/performance requirements cannot be directly mapped into open-loop system specifications. Recently, a robust controller design methodology was proposed using a numerical constrained optimization approach to maximize stability margins while meeting performance requirements ${ }^{5-9}$. This control design methodology has been successfully used to design a single robust control moment gyro (CMG) flex filter set for multiple International Space Station (ISS) stages $^{5}$ and robust flex filters and a PID controller for the Orbiter Repair Maneuver operation? The same design methodology for employing numerical optimization was developed for the Ares I bending filters, PID gains and anti-drift/load relief controller. The design objectives include attitude tracking accuracy, load relief, anti-drift and robust stability with respect to rigid body dynamics, propellant slosh, and flex ${ }^{10}$.

Under the assumption that the Morpheus time-varying dynamics and control system can be frozen over a short period of time, the bending filters are designed to stabilize all the selected frozen-time launch control systems in the 
presence of parametric uncertainty. Due to the rigid-slosh interaction, the slosh filter design achieves stability by phase stabilizing the slosh modes. Similarly, the Roll Control System was designed to deal with disturbance torques due to aerodynamics and vehicle thrust vector and mass properties dispersions while providing steering torque for roll control commanded by guidance. These effects had to be considered in the design of the phase planes to provide the required attitude performance while conserving propellant. A design methodology for employing numerical optimization ${ }^{5,10}$ was used for the Morpheus flight control system design.

The Morpheus flight control system designs presented here have been demonstrated to provide stable control systems in Draper Ascent vehicle Stability Analysis Tool (ASAT) ${ }^{11}$ and verified in the JSC Trick ${ }^{12}$ Morpheus time domain simulation and flight tests.

\section{Morpheus Flight Control System}

The cross coupling effect between axes in the Morpheus Lander vehicle is small. This enables design and analysis to be performed in each axis separately. The Morpheus attitude control system consists of both gimbal control and fixed jet reaction control. The former is used for pitch and yaw control while the latter is utilized for roll control. Due to the symmetry in the pitch and yaw axes as shown in Figure 2, the TVC control system can be designed for either the pitch or yaw axis ${ }^{13}$.

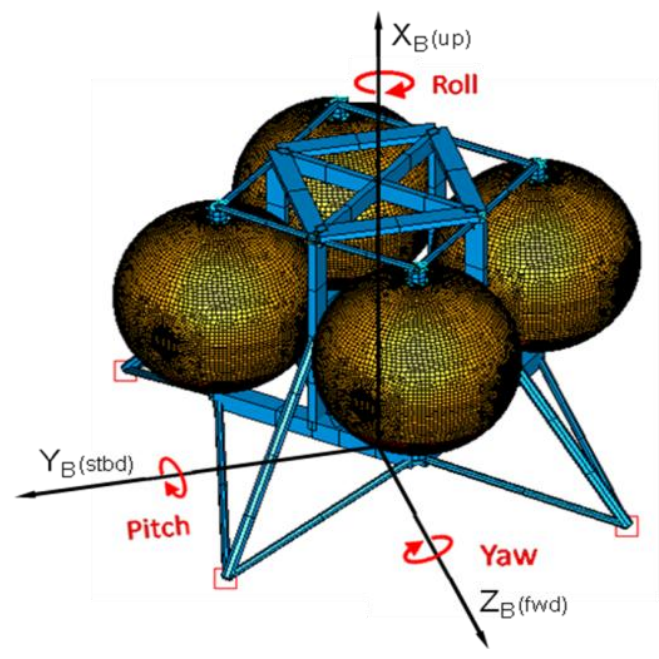

Figure 2. Morpheus Vehicle Coordinate Frame

\section{$\underline{\text { Lateral Control System }}$}

The 2 DOF equations presented in their entirety in Reference 13-15 can be divided into rigid body dynamics, flex (bending) dynamics, and slosh dynamics. Figure 3 shows the sign conventions for the equations of motion with variables as defined in the List of Variables ${ }^{13}$. Note that the 2 DOF in this depiction include translation in the Y-axis and rotation in the Yaw direction. Rigid body dynamics includes rigid rotation about the center of gravity and the rigid body translation of the center of gravity. Specifically,

$$
\phi_{c . g .} s^{2}=-c_{1} \alpha-c_{2} \beta_{E}-\frac{1}{I_{z z}}\left(X_{c . g} S_{E}+I_{E}\right) \beta_{E} s^{2}+\frac{1}{I_{z z}} \sum_{j=1}^{n_{s}} m_{s j}\left(l_{s j} s^{2}+k_{3}\right) Z_{s j}
$$

and

$$
Z_{c . g .} s^{2}=k_{7} \alpha+k_{4} \beta_{E}+k_{3} \phi_{c . g .}+\frac{S_{E}}{M} \beta_{E} s^{2}-\frac{1}{M} \sum_{j=1}^{n_{s}} m_{s j} Z_{s j} s^{2}
$$




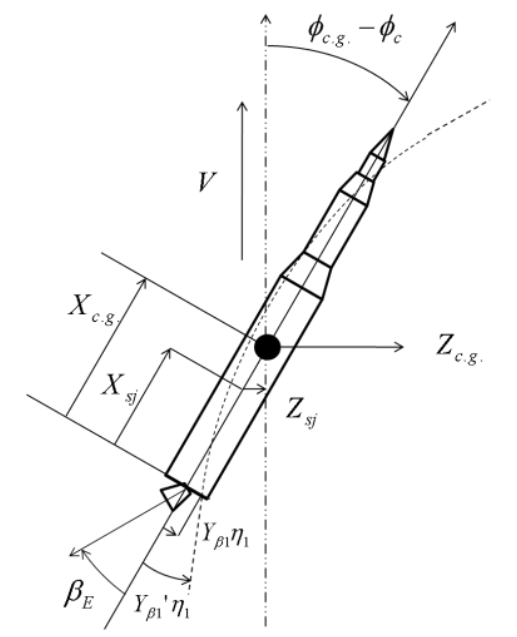

Figure 3. Spacecraft Sign Conventions

Rigid body motion depends on forces and torques created by aerodynamic pressure, gimbal angle (including the "tail-wags-dog" effect), thrust, drag, and sloshing fuel masses. Angle of attack for the undeformed vehicle is a function of wind velocity $\left(V_{w}\right)$, rigid attitude $\left(\phi_{c . g .}\right)$, and rigid velocity $\left(Z_{c . g . s}\right)$

$$
\alpha=\left(V_{w} / V\right)+\phi_{c . g .}-\left(Z_{c . g .} s / V\right)
$$

Bending motion depends on flexibility characteristics such as natural frequency, damping, and generalized mass in addition to the forces mentioned above for the rigid body dynamics. Specifically,

$$
\left(s^{2}+2 \xi_{b i} \omega_{b i} s+\omega_{b i}^{2}\right) M_{i} \eta_{i}=F Y_{\beta i} \beta_{E}+\left(S_{E} Y_{\beta i}-I_{E} Y_{\beta i}^{\prime}\right) \beta_{E} s^{2}-\sum_{j=1}^{n_{s}} m_{s j} Y_{s i j} Z_{s j} s^{2}+C_{Z \eta_{i}} \alpha
$$

Each bending mode is represented by a separate equation. The inclusion of more bending modes in a model leads to an increase in model fidelity. However, this also leads to an increase in the size and complexity of the system. The number of modes included in an analysis ultimately depends on the required model fidelity, the vehicle's frequency spectrum, and the available computation tools.

The dynamics of sloshing fuel is described by an equation for each fuel tank being considered in the analysis. Specifically,

$$
\left(s^{2}+2 \xi_{s j} \omega_{s j} s+\omega_{s j}^{2}\right) Z_{s j}=-Z_{c . g .} s^{2}+\left(l_{s j} s^{2}+k_{3}\right) \phi_{c . g .}-\sum_{i=1}^{n_{b}} Y_{s i j} \eta_{i} s^{2}
$$

Similar to bending modes, the inclusion of more slosh masses (up to the true number) yields a higher fidelity model. As shown in Equations 1, 2, and 4, the slosh tanks with greater slosh masses have a larger effect on overall vehicle motion. Equations 1 through 5 describe the overall yaw motion of the spacecraft and collectively form the plant dynamics.

In addition to the plant dynamics, the control system includes a combination of other systems designed to regulate the spacecraft's attitude. Sensors on the body of the vehicle determine the attitude and attitude rate according to the equations

$$
\phi_{P}=\phi_{c . g .}+\sum_{i=1}^{n_{b}} Y_{\phi i}^{\prime} \eta_{i}
$$

and

$$
\phi_{R G} s=\phi_{c . g .} s+\sum_{i=1}^{n_{b}} Y_{\phi i}^{\prime} \eta_{i} s
$$

respectively. It is apparent that sensor output only differs from plant output if bending dynamics are included in the model. Note that, when convenient, the sensor equations can be included as part of the plant's output equations. 
Sensor output is filtered in order to attenuate the higher frequency bending modes ${ }^{10,13,14}$. The filter output becomes the PID controller input. The PID controller outputs a gimbal command angle

$$
\beta_{C}=K_{I} \int\left(\phi_{P}-\phi_{C}\right)+K_{P}\left(\phi_{P}-\phi_{C}\right)+K_{D} \dot{\phi}_{R G}
$$

The controller gains $\left(K_{I}, K_{P}\right.$, and $\left.K_{D}\right)$ are designed using gain scheduling, a process that involves optimizing a controller for specific design points throughout the time of flight and linearly interpolating between these points to obtain gain values for any flight time ${ }^{10,13}$. The command angle translates the gimbal angle via the dynamics of the actuator $\left(G_{G D}\right)$

$$
\beta_{E}=G_{G D} \beta_{C}
$$

The lateral attitude control system model, as shown in Figure 4 includes the Morpheus dynamics, guidance and controller modules. The dynamics modules consist of both rigid and slosh dynamics models ${ }^{1}$. The dynamics modules take in actual gimbal angles and output attitude and rate gyro measurements. The rate signals are filtered by the rate filters. The attitude commands are generated from the steering block, which takes in the weighted sum of position and velocity tracking errors. The attitude and rate error signals represent the difference between commanded and actual attitude and rate. The error signals are sent to the PID controller to generate the commanded gimbal angles, which drive the gimbal dynamics.

\section{Rotational Motion Control}

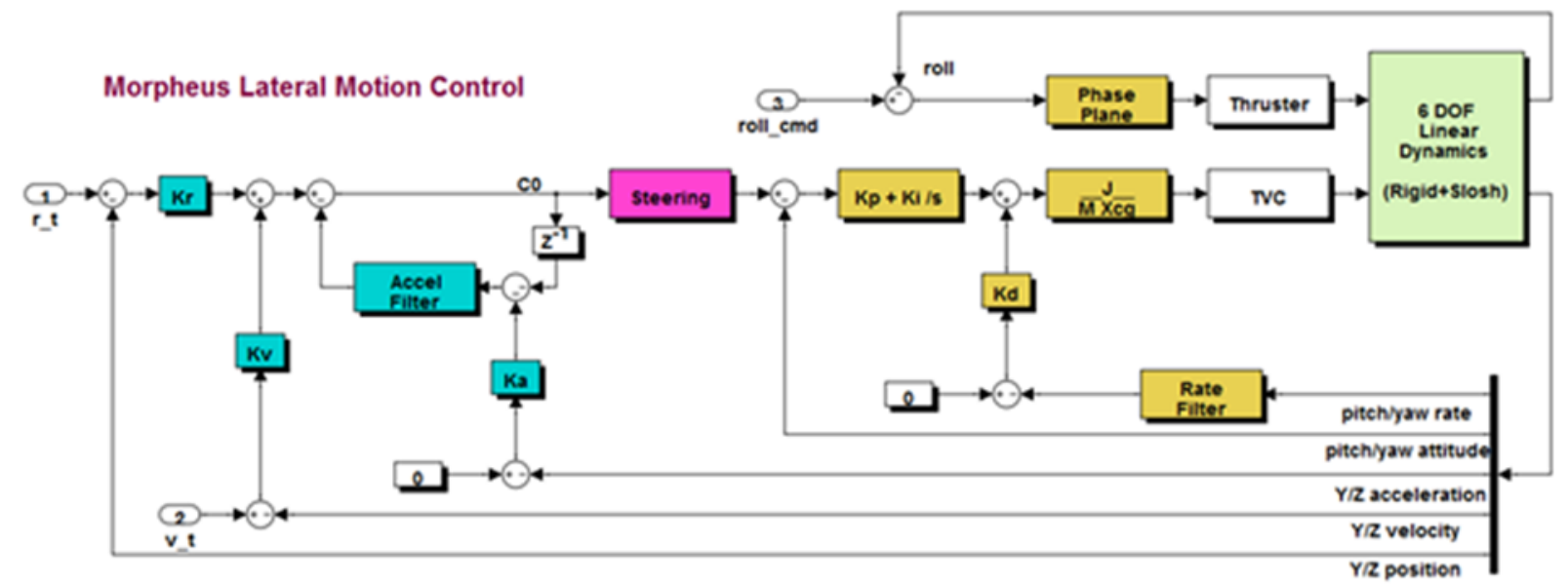

Figure 4. Morpheus Lateral Flight Control System Model

Both position and velocity measurements are fed back in the outer loop. The weighted position and velocity errors are summed to generate estimated acceleration commands. The steering algorithm takes in the estimated acceleration commands to generate attitude commands. To improve attitude pointing accuracy, both lateral Y/Z accelerations are fed back in the outer loop. The acceleration filters are designed to update the trimmed attitude command from the sensed acceleration, as well as eliminate the high frequency sensor noise shown in Figure 4.

\section{$\underline{\text { Roll Control System }}$}

The Morpheus flight control system uses phase plane autopilots for roll attitude control ${ }^{1}$. The roll phase plane autopilot produces a single command that takes on three states; 0 for drift, +1 for positive rotation and -1 for negative rotation. This command is sent to a jet selection logic that sets the appropriate fixed jet command(s) to "on" or "off."

Because the high-frequency flex dynamics possess the ability to make the system unstable, it is critical that a low pass filter be employed to attenuate high-frequency flex modes. The rigid and flex plant dynamics are integrated 
with the flex filter and phase plane controller to create the roll dynamics and control system. The block diagram in Figure 5 outlines the dynamics and control components.

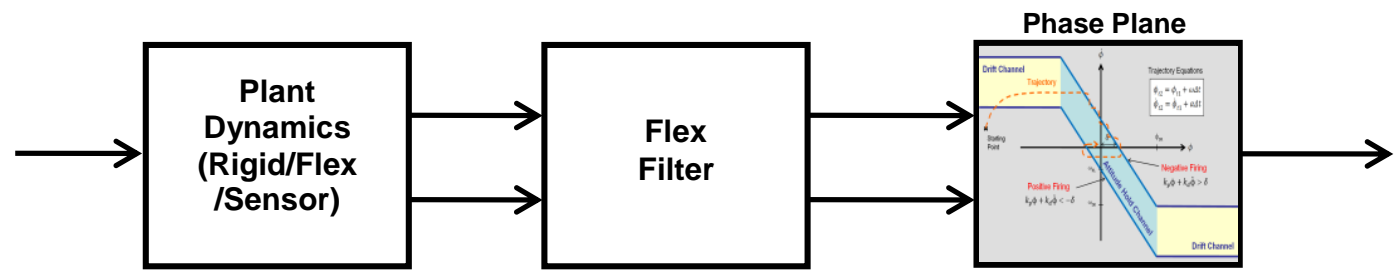

Figure 5. Roll Control System Model

The flex filter block consists of a set of low-pass filters to attenuate high-frequency noise while, at the same time, allowing low-frequency dynamics to feedback into the controller. A phase plane control system regulates attitude tracking and performance. Unlike the PID controller in the TVC control system, the phase plane controller is an inherently nonlinear system that necessitates the use of nonlinear techniques in order to predict the system's behavior. The phase plane controller is "an idealized method of treating performance optimization for classes of minimum time and/or minimum fuel problems" ${ }^{\prime \prime}$. The phase plane controller offers a unique method for attitude control while responding to the vehicle dynamics in the plane defined by state errors and state rate errors. The trajectories in the phase plane can be described through Equations 10 and $11^{17}$.

$$
\begin{aligned}
& \phi_{r 2}=\phi_{r 1}+\omega_{r} \Delta t \\
& \dot{\phi}_{r 2}=\dot{\phi}_{r 1}+\alpha_{r} \Delta t
\end{aligned}
$$

In the expressions above $\omega_{r}$ is the roll angular velocity, $\alpha_{r}$ is the roll angular acceleration and $\Delta t$ is the thruster firing time for the phase plane controller.

Figure 6 shows how trajectories in the phase plane operate. For example, consider the starting point in Figure 6. The system applies a continuous torque until it enters the drift channel. At this point, the thrusters discontinue their firing and the system's attitude continues to increase because the system is in the upper half of the phase plane, which means it has a positive rate. The rate is constant as long as there is no firing because the system cannot accelerate. Once the system crosses the negative switch line into the negative firing region, the system undergoes a negative acceleration which places the system back into the non-firing region; however, the system's attitude will continue to move towards the negative firing region until the system has been driven into the lower half of the phase plane. Once there, the system's attitude will decrease in the non-firing region until it crosses the positive switch line at which point a positive firing will occur ${ }^{17}$.

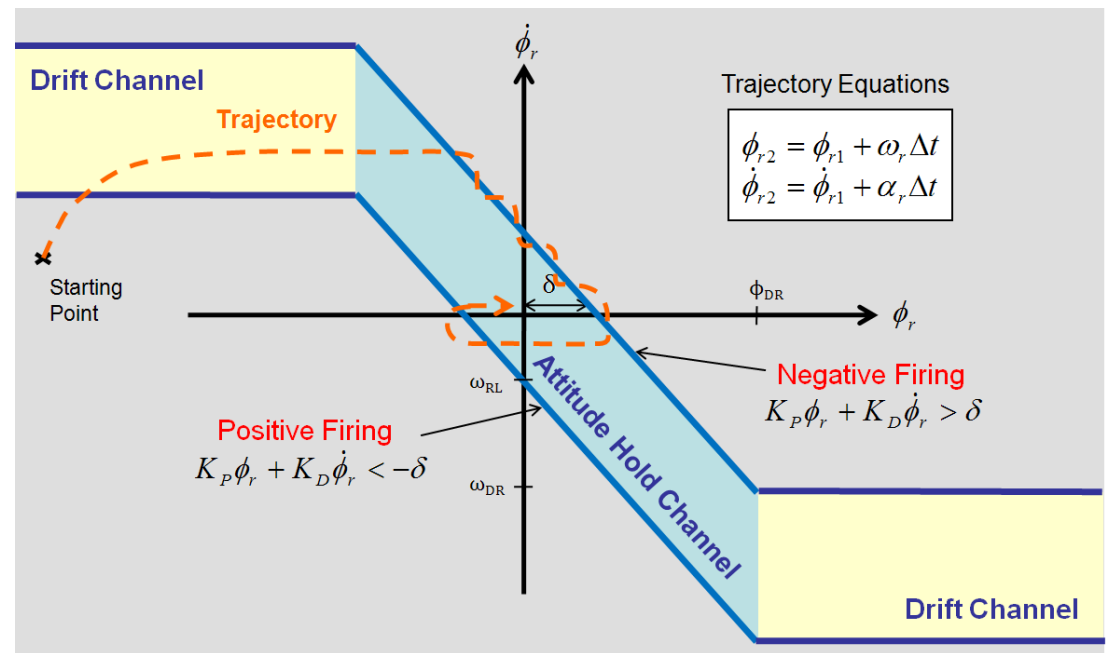

Figure 6. Simplified Phase Plane Controller 
The system will continue to oscillate around the origin of the phase plane in what is called a limit cycle. A common definition of a limit cycle is an oscillation of "fixed amplitude and fixed period without external excitation" $" 18$. In order to evaluate a nonlinear system such as a phase plane controlled system, it is necessary to transform the phase plane controller into a form where control techniques can be applied. This will be accomplished in a two-step process. First, only the attitude hold region will be evaluated, and second, the phase plane controller will be transformed into an equivalent system consisting of a PD controller and a nonlinear element consisting of a dead zone and an ideal relay. This procedure is illustrated in Figure 7. The two switching curves in Figure 6, which define the dead zone between the positive and negative firing regions, can be defined by the inequality:

$$
-\left(\omega_{R L} / \delta\right) \phi_{r}-\omega_{R L}<\dot{\phi}_{r}<-\left(\omega_{R L} / \delta\right) \phi_{r}+\omega_{R L}
$$

In order to proceed from step one to step two in Figure 7, it is necessary to implement a PD controller. It is first necessary to recast Equation 12 in the form

$$
-1<\phi_{r} / \delta+\dot{\phi}_{r} / \omega_{R L}<1
$$

Multiplying Equation 13 by the dead zone, $\delta$, leads to:

$$
-\delta<\phi_{r}+\left(\delta / \omega_{R L}\right) \dot{\phi}_{r}<\delta
$$

This inequality provides the following values for $K_{P}$ and $K_{D}$, which are the proportional and derivative gains respectively ${ }^{17}$.

$$
\begin{gathered}
K_{P}=1 \\
K_{D}=\delta / \omega_{R L}
\end{gathered}
$$

The $K_{P}$ and $K_{D}$ values calculated above are critical in creating a practical phase plane controller that can be used with control analysis techniques. Substituting these values into Equation 14 yields the result for the dead zone ${ }^{17}$.

$$
-\delta<K_{P} \phi_{r}+K_{D} \dot{\phi}_{r}<\delta
$$

It has been demonstrated in Reference 19 that with the above approximation, both Pulse-Width Modulation (PWM) and absolute stability theory can be used to predict the stability margin of a phase plane control system. The applications of both techniques have been extended to analyze and design the Morpheus roll control system ${ }^{19}$.
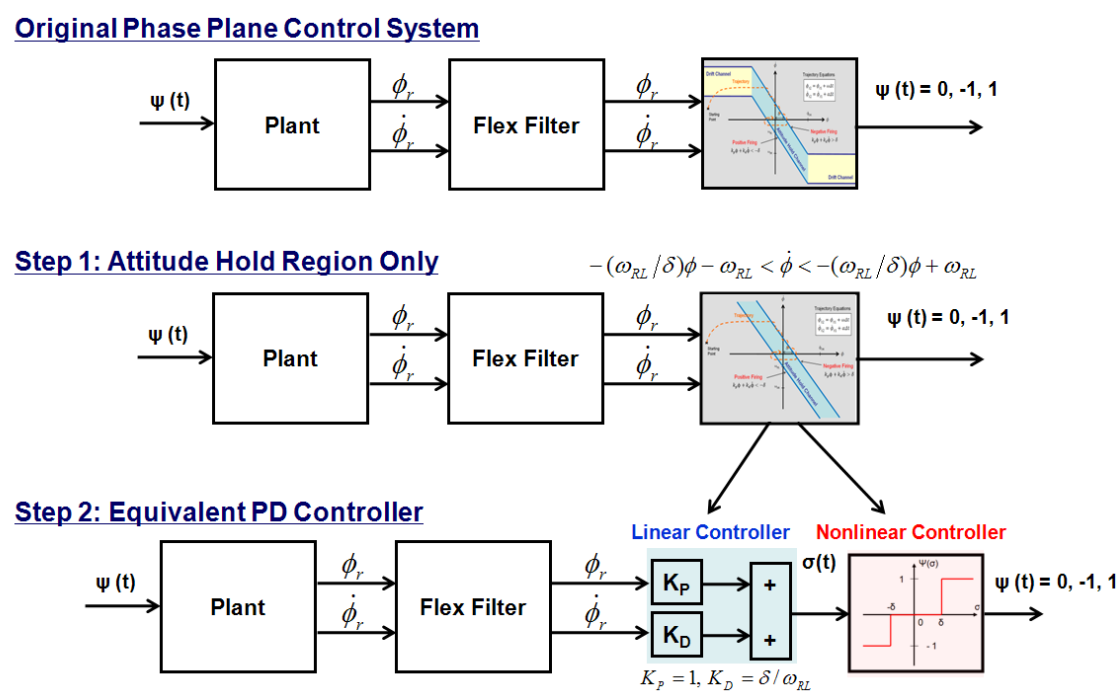

Figure 7. PD-Equivalent Phase Plane Development

\section{Morpheus Vertical Control}

The Morpheus vertical flight control system can be modeled as shown in Figure 8. The guidance block takes in the measured and commanded vertical position and velocity data, and then generates an acceleration command, which is the weighted sum of the position and velocity errors. Both commanded and sensed vertical acceleration data then send to the throttle control to generate a delta throttle command to compensate for the unmodeled acceleration force. A first order transfer function is used to represent actuator dynamics. 


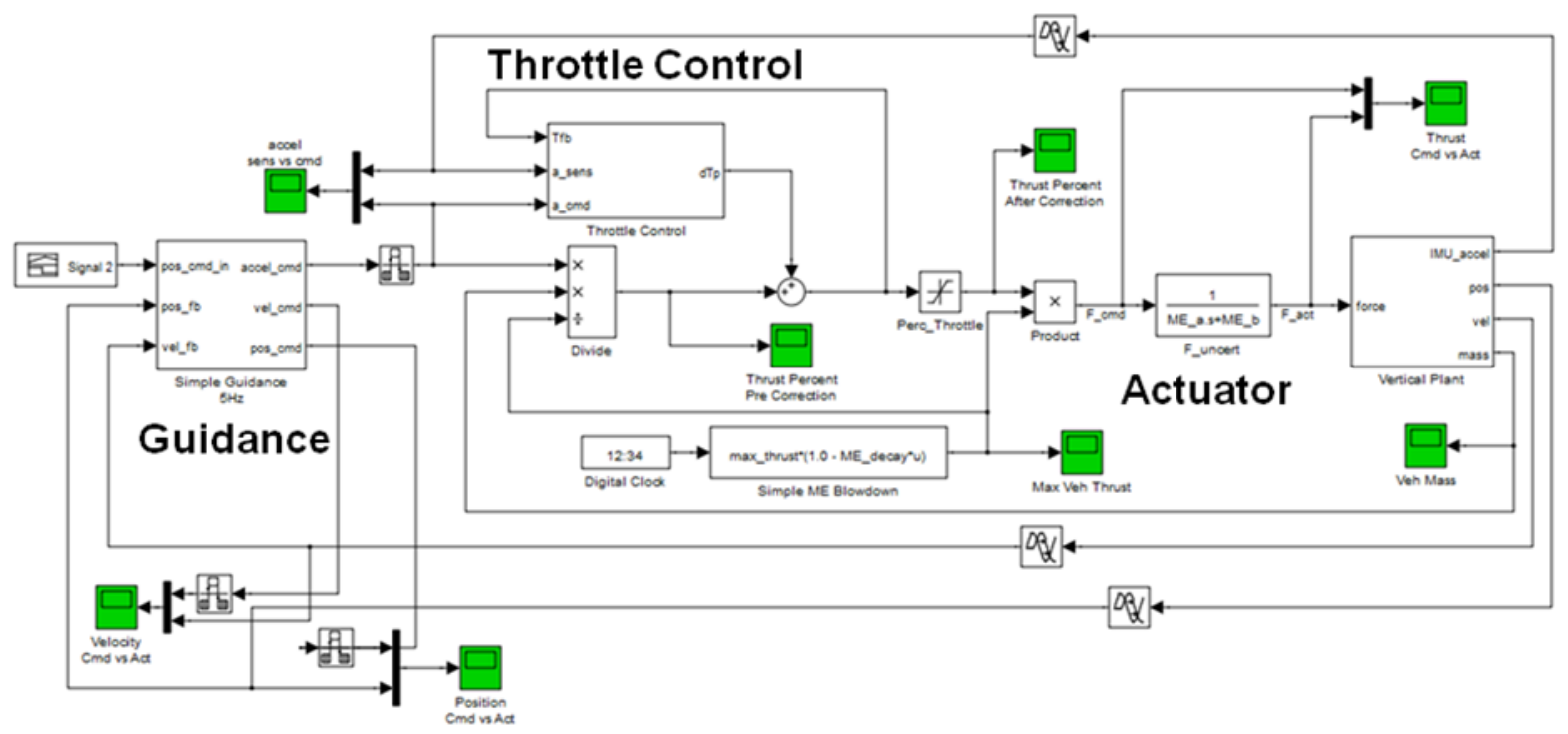

Figure 8. Morpheus Vertical Flight Control System Model

\section{Constrained Optimization Approach for Morpheus Flight Control System Design}

It has been previously demonstrated in multiple space applications ${ }^{5-11}$ that bending filters can be designed numerically using a constrained optimization framework. The design parameters are the coefficients of the bending filters. For example, if an $\mathrm{n}^{\text {th }}$ order transfer function architecture is selected for both the attitude and rate filter, the total number of design parameters is $4 \mathrm{n}$.

$$
\begin{aligned}
& F_{\text {attitude }}(s)=\prod_{i=0}^{N-1} \frac{s^{2}+2 x_{4 *_{i+2}} x_{4 *_{i+1}} s+x_{4 *_{i+1}}{ }^{2}}{s^{2}+2 x_{4 *_{i+4}} x_{4 *_{i+3}} s+x_{4 *_{i+3}}{ }^{2}} \\
& F_{\text {rate }}(s)=\prod_{i=0}^{N-1} \frac{s^{2}+2 x_{4 *_{i+2}} x_{4 *_{i+1}} s+x_{4 *_{i+1}}{ }^{2}}{s^{2}+2 x_{4 *_{i+4}} x_{4 *_{i+3}} s+x_{4 *_{i+3}}{ }^{2}}
\end{aligned}
$$

In addition to filter coefficients, PID and Guidance controller gains/filter coefficients are also included in the design parameters set. A set of feasible parameters must satisfy the following constraints:

(C1). The filter itself must be stable and minimal phase to guarantee stability and performance.

(C2). The bandwidth of the bending filter should be greater than that of the PID controller to avoid rigid performance degradation.

These constraints can be used to set the upper and lower bounds for the bending filter design.

The primary objective of Ares-I control system design is to provide sufficient stability margins in the presence of various parameter uncertainties while maintaining adequate system response. Experience has shown that the following design objectives similar to Reference 13 are adequate:

(O1). Both nominal and perturbed closed-loop Morpheus control system must be stable.

$(\mathrm{O} 2)$. At least $3 \mathrm{~dB} / 20$ degree robust rigid gain/phase margin is required for perturbed control system.

To ensure adequate response to guidance command, attitude step response specifications are imposed in the optimization problem. The additional constraints minimize the performance degradation due to the addition of the bending filters. These attitude pointing specifications, which include requirements for rise time, percent overshoot, settling time, and steady state errors, vary during the entire flight time. 
The Morpheus control system must also ultimately demonstrate robustness to uncertainties in the plant. The goal is to design bending filters that are robust to uncertainty in slosh mass/damping/frequency, vehicle mass properties, engine mass properties, aerodynamics characteristics and transportation latency. Once design objectives and constraints are identified, the bending filter design task is ready to be cast as the following constrained optimization problem

$$
\begin{array}{cc}
\min _{x} & f(x) \\
\text { s.t. } & \\
& g(x) \leq 0 \\
& x_{l} \leq x \leq x_{u}
\end{array}
$$

The filter design criteria (C1) and (C2) can be formulated as inequality constraints. These inequality constraints can be also cast as objectives $f(x)$ in the above multi-objective constrained optimization problem. In general, these objectives are competing with each other. For example, maximizing gain margins usually diminishes phase margins. Therefore, there is no unique solution to this problem. To address this, Pareto optimality ${ }^{20}$ must be applied to characterize the objectives. This is accomplished with a weighted sum strategy, which converts the multi-objective problem into a single objective optimization problem.

\section{Analysis of Morpheus Flight Control System Design}

The Morpheus flight control system consists of Thrust Vector Controllers (TVCs) using identical pitch/yaw autopilot architectures and a phase plane autopilot for roll control.

The thrust vector control law is PID with gains based on mass table lookup to accommodate changing flight conditions. The PID gains were designed for rigid body performance, but also to provide as much frequency separation as possible between the rigid body and slosh mode frequencies. The flex filters were designed to phase stabilize the slosh modes. Slosh dynamics are also damped passively using mechanical baffles in the tanks, and filtering in the control system is used to supply adequate slosh gain/phase margin for phase stabilized slosh modes when passive damping is inadequate for slosh gain stabilization.

The robustness of the Morpheus flight control system designs are verified in the linear frequency analysis using Draper Ascent vehicle Stability Analysis Tool (ASAT) for all dispersed designated trajectories. An ASAT utility imports each nominal datum and identifies the correct slosh mass property for each of a series of frozen time points. The analysis time vector is the same for each trajectory and is chosen to provide snapshots of flight with sufficient control authority (thrust magnitude) such that the stability analysis is valid. The margin calculation tool determines the characteristics of the response and tracks both the worst-case margin values and the seed identifiers that created the worst-case systems such that they can be later replicated. The following margins and characteristics are identified if they exist:

Rigid gain margin: minimum gain margin at rigid phase crossover frequencies.

Rigid phase margin: minimum phase margin at rigid gain crossover frequency.

Rigid slosh margin: minimum gain margin at slosh phase crossover frequencies.

The relevant characteristics are illustrated on the Nichols response shown in Figure 10. The robust frequency margin requirements for the current design are listed in $(\mathrm{O} 2)$.

An example of a stability margin evaluation of the nominal and dispersed control system using ASAT analysis are summarized for both the vertical control system (Figure 9(a)) and the lateral control system (Figure 9(b)). The design goal is to ensure that the Morpheus flight control system possess sufficiently robust stability gain and phase margins for the entire flight region of multiple planned missions.

After the flight control system is designed and analyzed in the frequency domain, a high fidelity nonlinear Trick simulation is used to verify stability and performance for the Morpheus control system. A Monte Carlo analysis is performed by perturbing system parameters such as mass property, center of mass location, thruster misalignment, 
as well as biased wind to evaluate the performance and robustness of the Morpheus flight control system. A sample Monte Carlo Analysis result is illustrated in Figure 11.

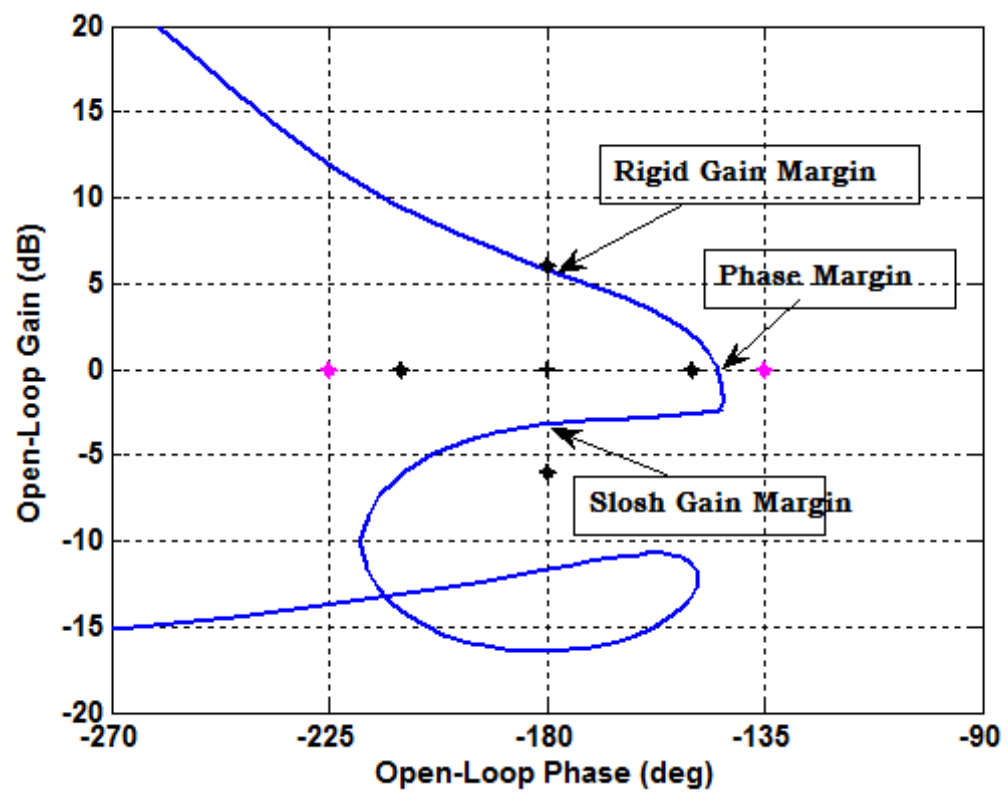

Figure 10. Stability Margin Definition Examples
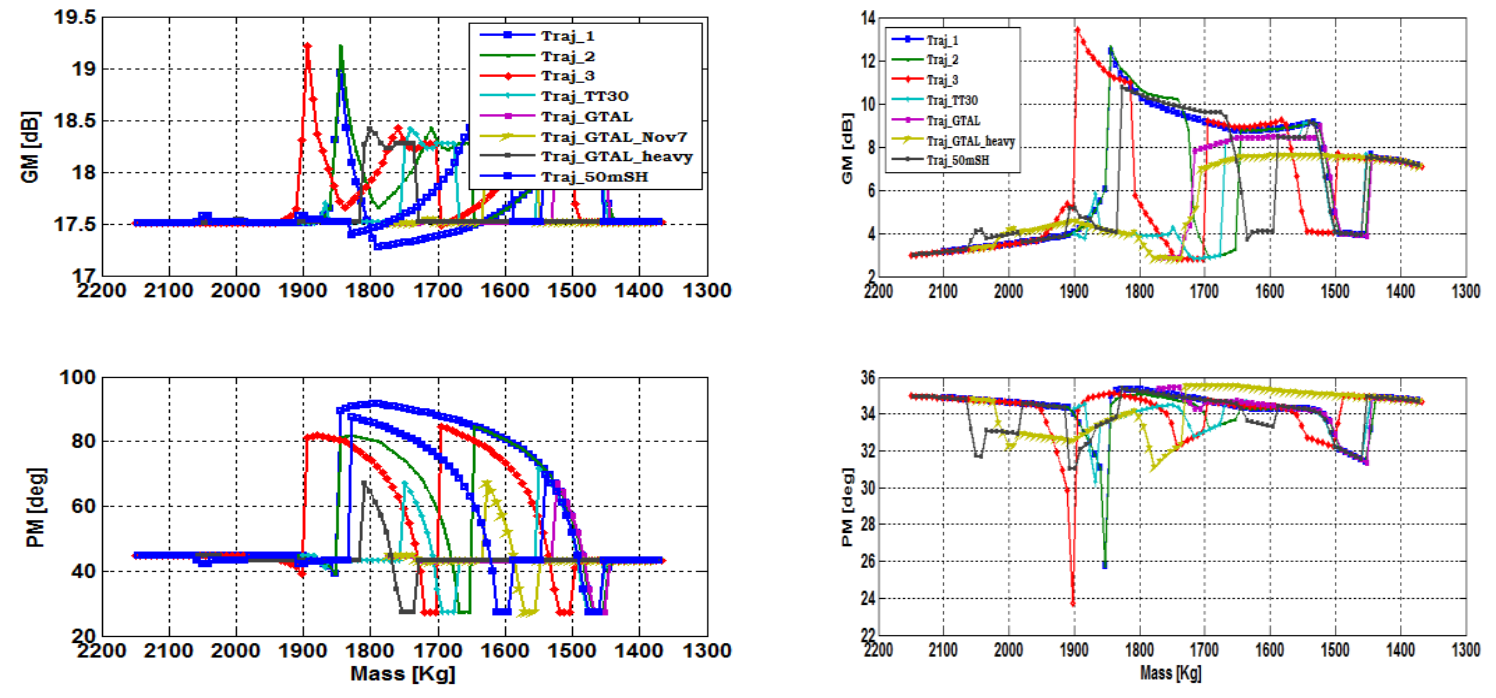

(a)

(b)

Figure 9. Morpheus ASAT Stability Analysis Example 


\section{Monte Carlo Truth Trajectories}

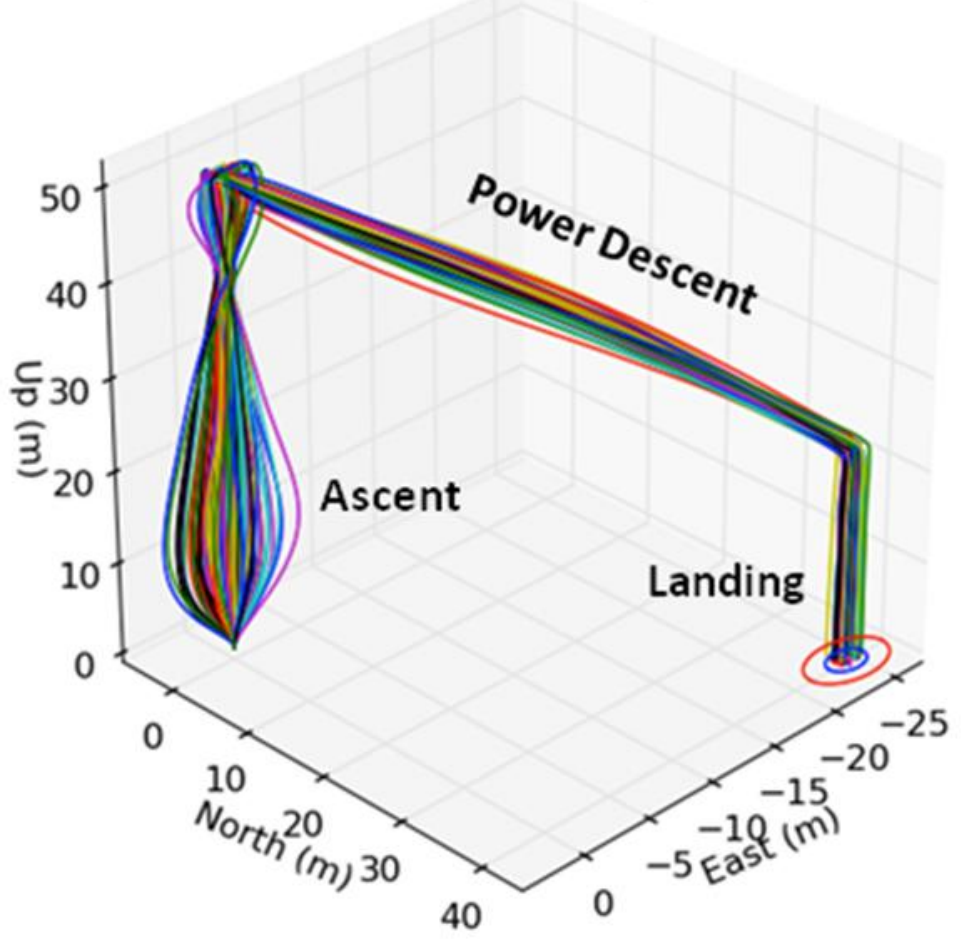

Figure 11. Morpheus Trick Monte Carlo Analysis Example

\section{Summary}

The Morpheus Lander is a vertical takeoff and landing test bed vehicle developed to demonstrate the system performance of the GN\&C system capability for the integrated autonomous landing and hazard avoidance system hardware and software. This paper presents a design methodology employing numerical optimization to develop the Morpheus flight control system. The flight control system design methodology was based on a numerical constrained optimization approach to maximize performance while meeting stability margins requirements. The flight control system designs provided herein have been demonstrated to provide stable flight control system in Draper ASAT, NASA/JSC Trick Morpheus time domain simulation, and flight tests.

\section{References}

1 Olansen, J. B, Munday, S. R., and Mitchell, J. D., and Baine, M., "Morpheus: Advancing Technologies for Human Exploration," Global Exploration Conference, CLEX-2012.05.2.4.x12761, 2012.

2 Oppenheim, A. V, and Schafer, R. W., Digital Signal Processing, Prentice-Hall Company, Inc., Englewood Cliffs, New Jersey, 1975.

3 Friedlander, B., and Porat, B, “The Modified Yule-Walker Method of ARMA Spectral Estimation," IEEE Transactions on Aerospace Electronic Systems, AES-20, No. 2, March 1984, pp. 158-173.

${ }^{4}$ Strum, R. D. and Kirk, D. E., First Principles of Discrete Systems and Digital Signal Processing, Addison-Wesley Publishing Company, Inc., New York, April 1989.

5 Jang, J., Bedrossian, N., Lee, A, Spanos, P., “A Constrained Optimization Approach for CMG Robust Flex Filter Design”, AIAA GN\&C Conference, August 2002.

${ }^{6}$ Jang, J., Lee, A., Bedrossian, N., Spanos, P., "Design of Robust Nash Game Theoretic Controllers with Time Domain Constraints," American Control Conference, 2003.

7 Bedrossian, N., Jang, J., Alaniz, A., Johnson, M., Sebelius, K., Mesfin, Y., "International Space Station US GN\&C Attitude Hold Controller Design for Orbiter Repair Maneuver,” AIAA GN\&C Conference, August, 2005. 
${ }^{8}$ Postma, B., Jang, J., Bedrossian, N., Spanos, P., "Robust Constrained Optimization Approach for International Space Station Centrifuge Rotor Auto-Balancing Controller," AIAA GN\&C Conference, August, 2005.

9 Jang, J., Bedrossian, N., Hall, R., Norris, L. H., Hall, C., Jackson, M., "Initial Ares-I Bending Filter Design," $30^{\text {th }}$ AAS Guidance and Control Conference, AAS 07-078, Breckenridge, Colorado, February 2007.

${ }^{10}$ Jang, J., Hall, R., Bedrossian, N., and Hall, C., "Ares-I Bending Filter Design Using A Constrained Optimization Approach," AIAA GN\&C Conference, Honolulu, HI, 2008.

${ }^{11}$ Jang, J., Alaniz, A., Hall, R., and Bedrossian, N., Hall, C., and Jackson, M., "Design of Launch Vehicle Control Systems Using Ascent Vehicle Stability Analysis Tool," AIAA Guidance and Control Conference, Portland, Oregon, Aug., 2011.

${ }^{12}$ Paddock, E. J., Lin, A., Vetter, K., and Crues, E. Z., “Trick: A Simulation Development Toolkit," AIAA Modeling and Simulation Technologies Conference and Exhibit, Austin, TX, 2003.

${ }^{13}$ Frosch, J.A., Vallely, D.P., "Saturn AS-501/S-IC Flight Control System Design”, Journal of Spacecraft, Vol. 4, No. 8, August 1967

${ }^{14}$ Haussermann, W., Duncan, R.C, "Status of Guidance and Control Methods, Instrumentation, and Techniques as Applied to the Apollo Project", Agardograph 92, NASA Code ATSS-AD, October 1964.

${ }^{15}$ Greensite, A. L., Analysis and Design of Space Vehicle Flight Control Systems, Spartan Book, New York, 1970.

${ }^{16}$ Penchuk, A., Hattis, P., and Kubiak, E., "A Frequency Domain Stability Analysis of a Phase Plane Control System," Journal of Guidance, Vol. 8, No. 1, 1984

${ }^{17}$ Wertz, J., Spacecraft Attitude Determination and Control, Boston, MS: D. Reidel Publishing Company, 1995.

${ }^{18}$ Gelb, A., and Velde, W. V., Multiple-Input Describing Functions and Nonlinear System Design, New York, NY: McGrawHill Book Co., 1968.

${ }^{19}$ Jang, J., Plummer, M., Bedrossian, N., Hall, C., Jackson, M., and Spanos, P., “Absolute Stability Analysis of a Phase Plane Controlled Spacecraft," 20th AAS/AIAA Space Flight Mechanics Meeting, AAS 10-112, San Diego, CA, February 2010.

${ }^{20}$ Rao, S. S., "Game Theory Approach for Multi-objective Structural Optimization," Computers \& Structures, Vol. 24, No. 1, 1987, pp. 119-127. 